\title{
Finite-Time Stability of Fractional-Order BAM Neural Networks with Distributed Delay
}

\author{
Yuping Cao ${ }^{1}$ and Chuanzhi Bai ${ }^{2}$ \\ ${ }^{1}$ Department of Basic Courses, Lianyungang Technical College, Lianyungang, Jiangsu 222000, China \\ ${ }^{2}$ Department of Mathematics, Huaiyin Normal University, Huaian, Jiangsu 223300, China \\ Correspondence should be addressed to Chuanzhi Bai; czbai@hytc.edu.cn
}

Received 8 February 2014; Accepted 1 April 2014; Published 22 April 2014

Academic Editor: Sabri Arik

Copyright (c) 2014 Y. Cao and C. Bai. This is an open access article distributed under the Creative Commons Attribution License, which permits unrestricted use, distribution, and reproduction in any medium, provided the original work is properly cited.

Based on the theory of fractional calculus, the generalized Gronwall inequality and estimates of mittag-Leffer functions, the finitetime stability of Caputo fractional-order BAM neural networks with distributed delay is investigated in this paper. An illustrative example is also given to demonstrate the effectiveness of the obtained result.

\section{Introduction}

Fractional calculus (integral and differential operations of noninteger order) was firstly introduced 300 years ago. Due to lack of application background and the complexity, it did not attract much attention for a long time. In recent decades fractional calculus is applied to physics, applied mathematics, and engineering [1-6]. Since the fractionalorder derivative is nonlocal and has weakly singular kernels, it provides an excellent instrument for the description of memory and hereditary properties of dynamical processes. Nowadays, study on the complex dynamical behaviors of fractional-order systems has become a very hot research topic.

We know that the next state of a system not only depends upon its current state but also upon its history information. Since a model described by fractional-order equations possesses memory, it is precise to describe the states of neurons. Moreover, the superiority of the Caputo's fractional derivative is that the initial conditions for fractional differential equations with Caputo derivatives take on the similar form as those for integer-order differentiation. Therefore, it is necessary and interesting to study fractional-order neural networks both in theory and in applications.

Recently, fractional-order neural networks have been presented and designed to distinguish the classical integerorder models [7-10]. Currently, some excellent results about fractional-order neural networks have been investigated, such as Kaslik and Sivasundaram [11, 12], Zhang et al. [13], Delavari et al. [14], and Li et al. [15, 16]. On the other hand, time delay is one of the inevitable problems on the stability of dynamical systems in the real word [17-20]. But till now, there are few results on the problems for fractional-order delayed neural networks; Chen et al. [21] studied the uniform stability for a class of fractional-order neural networks with constant delay by the analytical approach; Wu et al. [22] investigated the finite-time stability of fractional-order neural networks with delay by the generalized Gronwall inequality and estimates of Mittag-Leffler functions; Alofi et al. [23] discussed the finite-time stability of Caputo fractional-order neural networks with distributed delay.

The integer-order bidirectional associative memory (BAM) model known as an extension of the unidirectional autoassociator of Hopfield [24] was first introduced by Kosko [25]. This neural network has been widely studied due to its promising potential for applications in pattern recognition and automatic control. In recent years, integer-order BAM neural networks have been extensively studied [26-29]. However, to the best of our knowledge, there is no effort being made in the literature to study the finite-time stability of fractional-order BAM neural networks so far.

Motivated by the above-mentioned works, we were devoted to establishing the finite-time stability of Caputo fractional-order BAM neural networks with distributed 
delay. In this paper, we will apply Laplace transform, generalized Gronwall inequality, and estimates of Mittag-Leffler functions to establish the finite-time stability criterion of fractional-order distributed delayed BAM neural networks.

This paper is organized as follows. In Section 2, some definitions and lemmas of fractional differential and integral calculus are given and the fractional-order BAM neural networks are presented. A criterion for finite-time stability of fractional-order BAM neural networks with distributed delay is obtained in Section 3. Finally, the effectiveness and feasibility of the theoretical result is shown by an example in Section 4.

\section{Preliminaries}

For the convenience of the reader, we first briefly recall some definitions of fractional calculus; for more details, see $[1,2,5]$, for example.

Definition 1. The Riemann-Liouville fractional integral of order $\alpha>0$ of a function $u:(0, \infty) \rightarrow R$ is given by

$$
I_{0^{+}}^{\alpha} u(t)=\frac{1}{\Gamma(\alpha)} \int_{0}^{t}(t-s)^{\alpha-1} u(s) d s
$$

provided that the right side is pointwise defined on $(0, \infty)$, where $\Gamma(\cdot)$ is the Gamma function.

Definition 2. The Caputo fractional derivative of order $\gamma>0$ of a function $u:(0, \infty) \rightarrow R$ can be written as

$$
\begin{array}{r}
{ }_{0}^{C} D_{t}^{\gamma} u(t)=\frac{1}{\Gamma(n-\gamma)} \int_{0}^{t} \frac{u^{(n)}(s)}{(t-s)^{\gamma+1-n}} d s, \\
n-1<\gamma<n .
\end{array}
$$

Definition 3. The Mittag-Leffler function in two parameters is defined as

$$
E_{\alpha, \beta}(z)=\sum_{k=0}^{\infty} \frac{z^{k}}{\Gamma(k \alpha+\beta)},
$$

where $\alpha>0, \beta>0$, and $z \in \mathbb{C}$, where $\mathbb{C}$ denotes the complex plane. In particular, for $\beta=1$, one has

$$
E_{\alpha, 1}(z)=E_{\alpha}(z)=\sum_{k=0}^{\infty} \frac{z^{k}}{\Gamma(k \alpha+1)} .
$$

The Laplace transform of Mittag-Leffler function is

$$
\begin{array}{r}
\mathscr{L}\left\{t^{\beta-1} E_{\alpha, \beta}\left(-\lambda t^{\alpha}\right)\right\}=\frac{s^{\alpha-\beta}}{s^{\alpha}+\lambda}, \\
\left(\mathscr{R}(s)>|\lambda|^{1 / \alpha}\right),
\end{array}
$$

where $t$ and $s$ are, respectively, the variables in the time domain and Laplace domain and $\mathscr{L}\{\cdot\}$ stands for the Laplace transform.
In this paper, we are interested in the finite-time stability of fractional-order BAM neural networks with distributed delay by the following state equations:

$$
\begin{aligned}
&{ }_{0}^{C} D_{t}^{\alpha} x_{i}(t)=-c_{i} x_{i}(t)+\sum_{j=1}^{n} b_{i j} f_{j}\left(y_{j}(t)\right) \\
&+\sum_{j=1}^{n} \int_{0}^{\tau} r_{i j}(s) f_{j}\left(y_{j}(t-s)\right) d s+I_{i}, \\
& t \geq 0, \\
&{ }_{0}^{C} D_{t}^{\beta} y_{j}(t)=-c_{j} y_{j}(t)+\sum_{i=1}^{n} d_{j i} g_{\mathrm{i}}\left(x_{i}(t)\right) \\
&+\sum_{i=1}^{n} \int_{0}^{\tau} p_{j i}(s) g_{i}\left(x_{i}(t-s)\right) d s+\bar{I}_{j},
\end{aligned}
$$

or in the matrix-vector notation

$$
\begin{aligned}
{ }_{0}^{C} D_{t}^{\alpha} x(t)= & -C x(t)+B f(y(t)) \\
& +\int_{0}^{\tau} R(s) f(y(t-s)) d s+I, \\
{ }_{0}^{C} D_{t}^{\beta} y(t)= & -C y(t)+D g(x(t)) \\
& +\int_{0}^{\tau} P(s) g(x(t-s)) d s+\bar{I}, \quad t \geq 0,
\end{aligned}
$$

where $1<\alpha, \beta<2$. The model (6) is made up of two neural fields $F_{x}$ and $F_{y}$, where $x_{i}(t)$ and $y_{j}(t)$ are the activations of the $i$ th neuron in $F_{x}$ and the $j$ th neuron in $F_{y}$, respectively;

$$
(x(t), y(t))=\left(x_{1}(t), \ldots, x_{n}(t), y_{1}(t), \ldots, y_{n}(t)\right)^{T} \in \mathbb{R}^{2 n}
$$

is the state vector of the network at time $t$; the functions

$$
\begin{gathered}
f(y(t))=\left(f_{1}\left(y_{1}(t)\right), f_{2}\left(y_{2}(t)\right), \ldots, f_{n}\left(y_{n}(t)\right)\right)^{T}, \\
g(x(t))=\left(g_{1}\left(x_{1}(t)\right), g_{2}\left(x_{2}(t)\right), \ldots, g_{n}\left(x_{n}(t)\right)\right)^{T}
\end{gathered}
$$

are the activation functions of the neurons at time $t ; C=$ $\operatorname{diag}\left(c_{i}\right)$ is a diagonal matrix; $c_{i}>0$ represents the rate with which the $i$ th unit will reset its potential to the resting state in isolation when disconnected from the network and external inputs; $B=\left(b_{i j}\right)_{n \times n}$ and $D=\left(d_{j i}\right)_{n \times n}$ are the feedback matrix; $\tau>0$ denotes the maximum possible transmission delay from neuron to another; $R=\left(r_{i j}\right)_{n \times n}$ and $P=\left(p_{j i}\right)_{n \times n}$ are the delayed feedback matrix; $I=\left(I_{1}, \ldots, I_{n}\right)^{T}$ and $\bar{I}=$ $\left(\bar{I}_{1}, \ldots, \bar{I}_{n}\right)^{T}$ are two external bias vectors.

Let $\mathbb{C}^{1}\left([-\tau, 0], \mathbb{R}^{n}\right)$ be the Banach space of all continuously differential functions over a time interval of length $\tau$, 
mapping the interval $[-\tau, 0]$ into $\mathbb{R}^{n}$ with the norm defined as follows: for every $\varphi \in \mathbb{C}^{1}\left([-\tau, 0], \mathbb{R}^{n}\right)$,

$$
\begin{aligned}
\|\varphi\|_{1} & =\max \left\{\|\varphi\|,\left\|\varphi^{\prime}\right\|\right\} \\
& =\max \left\{\sup _{\theta \in[-\tau, 0]}|\varphi(\theta)|, \sup _{\theta \in[-\tau, 0]}\left|\varphi^{\prime}(\theta)\right|\right\} .
\end{aligned}
$$

The initial conditions associated with (6) are given by

$$
\begin{gathered}
x_{i}(\theta)=\varphi_{i}(\theta), \quad x_{i}^{\prime}(\theta)=\varphi_{i}^{\prime}(\theta), \quad y_{j}(\theta)=\psi_{j}(\theta), \\
y_{j}^{\prime}(\theta)=\psi_{j}^{\prime}(\theta), \quad \theta \in[-\tau, 0],
\end{gathered}
$$

where $\varphi_{i}, \psi_{j} \in C^{1}([-\tau, 0], \mathbb{R})$.

In order to obtain main result, we make the following assumptions.

(H1) For $i, j=1, \ldots, n$, the functions $r_{i j}(\cdot)$ and $p_{j i}(\cdot)$ are continuous on $[0, \tau]$.

(H2) The neurons activation functions $f_{i}$ and $g_{j}(i, j=$ $1, \ldots, n)$ are bounded.

(H3) The neurons activation functions $f_{i}$ and $g_{j}$ are Lipschitz continuous; that is, there exist positive constants $h_{i}, l_{j}(i, j=1, \ldots, n)$ such that

$\left|f_{i}(u)-f_{i}(v)\right| \leq h_{i}|u-v|, \quad\left|g_{j}(u)-g_{j}(v)\right| \leq l_{j}|u-v|$,

$\forall u, v \in \mathbb{R}$.

Since the Caputo's fractional derivative of a constant is equal to zero, the equilibrium point of system (6) is a constant vector $\left(x^{*}, y^{*}\right)=\left(x_{1}^{*}, x_{2}^{*}, \ldots, x_{n}^{*}, y_{1}^{*}, y_{2}^{*}, \ldots, y_{n}^{*}\right)^{T} \in \mathbb{R}^{2 n}$ which satisfies the system

$$
\begin{array}{r}
c_{i} x_{i}^{*}-\sum_{j=1}^{n} b_{i j} f_{j}\left(y_{j}^{*}\right)-\sum_{j=1}^{n} \int_{0}^{\tau} r_{i j}(s) f_{j}\left(y_{j}^{*}\right) d s-I_{i}=0, \\
i=1, \ldots, n, \\
c_{j} y_{j}^{*}-\sum_{i=1}^{n} d_{j i} g_{i}\left(x_{i}^{*}\right)-\sum_{i=1}^{n} \int_{0}^{\tau} p_{j i}(s) g_{i}\left(x_{i}^{*}\right) d s-\bar{I}_{j}=0, \\
j=1, \ldots, n .
\end{array}
$$

By using the Schauder fixed point theorem and assumptions (H1)-(H3), it is easy to prove that the equilibrium points of system (6) exist. We can shift the equilibrium point of system (6) to the origin. Denoting

$$
\begin{aligned}
(u(t), v(t))= & \left(u_{1}(t), \ldots, u_{n}(t), v_{1}(t), \ldots, v_{n}(t)\right)^{T} \\
= & \left(x_{1}(t)-x_{1}^{*}, \ldots, x_{n}(t)\right. \\
& \left.\quad-x_{n}^{*}, y_{1}(t)-y_{1}^{*}, \ldots, y_{n}(t)-y_{n}^{*}\right)^{T},
\end{aligned}
$$

then system (6) can be written as

$$
\begin{aligned}
{ }_{0}^{C} D_{t}^{\alpha} u_{i}(t)= & -c_{i} u_{i}(t)+\sum_{j=1}^{n} b_{i j} F_{j}\left(v_{j}(t)\right) \\
& +\sum_{j=1}^{n} \int_{0}^{\tau} r_{i j}(s) F_{j}\left(v_{j}(t-s)\right) d s,
\end{aligned}
$$

$t \geq 0$,

$$
\begin{array}{r}
{ }_{0}^{C} D_{t}^{\beta} v_{j}(t)=-c_{j} v_{j}(t)+\sum_{i=1}^{n} d_{j i} G_{i}\left(u_{i}(t)\right) \\
+\sum_{i=1}^{n} \int_{0}^{\tau} p_{j i}(s) G_{i}\left(u_{i}(t-s)\right) d s, \\
i, j=1, \ldots, n,
\end{array}
$$

with the initial conditions

$$
\begin{aligned}
& u_{i}(\theta)=\bar{\varphi}_{i}(\theta), \quad u_{i}^{\prime}(\theta)=\bar{\varphi}_{i}^{\prime}(\theta), \quad v_{j}(\theta)=\bar{\psi}_{j}(\theta), \\
& v_{j}^{\prime}(\theta)=\bar{\psi}_{j}^{\prime}(\theta), \quad \theta \in[-\tau, 0],
\end{aligned}
$$

where

$$
\begin{array}{r}
F_{j}\left(v_{j}(t)\right)=f_{j}\left(v_{j}(t)+y_{j}^{*}\right)-f_{j}\left(y_{j}^{*}\right), \\
G_{i}\left(u_{i}(t)\right)=g_{i}\left(u_{i}(t)+x_{i}^{*}\right)-g_{i}\left(x_{i}^{*}\right), \\
\bar{\varphi}_{i}(\theta)=\varphi_{i}(\theta)-x_{i}^{*}, \quad \bar{\psi}_{j}(\theta)=\psi_{j}(\theta)-y_{j}^{*}, \\
\theta \in[\tau, 0] .
\end{array}
$$

Similarly, by using the matrix-vector notation, system (15) can be expressed as

$$
\begin{aligned}
&{ }_{0}^{C} D_{t}^{\alpha} u(t)=-C u(t)+B F(v(t)) \\
&+\int_{0}^{\tau} R(s) F(v(t-s)) d s, \\
& t \geq 0, \\
&{ }_{0}^{C} D_{t}^{\beta} v(t)=-C v(t)+D G(u(t)) \\
&+\int_{0}^{\tau} P(s) G(u(t-s)) d s, \\
& t \geq 0,
\end{aligned}
$$

with the initial condition

$$
\begin{array}{ll}
u(\theta) & =\bar{\varphi}(\theta), \quad u^{\prime}(\theta)=\bar{\varphi}^{\prime}(\theta), \quad v(\theta)=\bar{\psi}(\theta), \\
v^{\prime}(\theta)=\bar{\psi}^{\prime}(\theta), & \theta \in[-\tau, 0],
\end{array}
$$

where

$$
\begin{gathered}
F(v(t))=\left(F_{1}\left(v_{1}(t)\right), F_{2}\left(v_{2}(t)\right), \ldots, F_{n}\left(v_{n}(t)\right)\right)^{T}, \\
G(u(t))=\left(G_{1}\left(u_{1}(t)\right), G_{2}\left(u_{2}(t)\right), \ldots, G_{n}\left(u_{n}(t)\right)\right)^{T} .
\end{gathered}
$$


Define the functions as follows:

$$
\begin{aligned}
& h_{i}(t)= \begin{cases}\frac{F_{i}\left(v_{i}(t)\right)}{v_{i}(t)}, & v_{i}(t) \neq 0, \\
0, & v_{i}(t)=0,\end{cases} \\
& l_{j}(t)= \begin{cases}\frac{G_{j}\left(u_{j}(t)\right)}{u_{j}(t)}, & u_{j}(t) \neq 0, \\
0, & u_{j}(t)=0,\end{cases}
\end{aligned}
$$

where $i, j=1, \ldots, n$. From assumption (H3), we can obtain $\left|h_{i}(t)\right| \leq h_{i},\left|l_{j}(t)\right| \leq l_{j}$. By (21), we have

$$
\begin{array}{r}
F_{i}\left(v_{i}(t)\right)=h_{i}(t) v_{i}(t), \quad G_{j}\left(u_{j}(t)\right)=l_{j}(t) u_{j}(t), \\
i, j=1, \ldots, n .
\end{array}
$$

Thus, system (18) can be further written as the following form:

$$
\begin{aligned}
&{ }_{0}^{C} D_{t}^{\alpha} u(t)=-C u(t)+B H(t) v(t) \\
&+\int_{0}^{\tau} R(s) H(t-s) v(t-s) d s, \\
& t \geq 0, \\
&+\int_{0}^{\tau} P(s) L(t-s) u(t-s) d s, \\
&{ }_{0}^{C} D_{t}^{\beta} v(t)=-C v(t)+D L(t) u(t) \\
& \quad t \geq 0,
\end{aligned}
$$

where $H(t)=\operatorname{diag}\left\{h_{i}(t)\right\}, L(t)=\operatorname{diag}\left\{l_{j}(t)\right\}$.

Definition 4. System (23) with the initial condition (19) is finite-time stable with respect to $\left\{\delta, \varepsilon, t_{0}, J\right\}, \delta<\varepsilon$, if and only if

$$
\|(\varphi, \psi)\|_{1}:=\|\varphi\|_{1}+\|\psi\|_{1}<\delta
$$

implies

$$
\|(u(t), v(t))\|=\|u(t)\|+\|v(t)\|<\varepsilon, \quad \forall t \in J,
$$

where $\delta$ is a positive real number and $\varepsilon>0, \delta<\varepsilon, t_{0}$ denotes the initial time of observation of the system, and $J$ denotes time interval $J=\left[t_{0}, t_{0}+T\right)$.

A technical result about norm upper-bounding function of the matrix function $E_{\alpha, \beta}$ is given in [30] as follows.

Lemma 5. If $\alpha \geq 1$, then, for $\beta=1,2, \alpha$, one has

$$
\left\|E_{\alpha, \beta}\left(A t^{\alpha}\right)\right\| \leq\left\|e^{A t^{\alpha}}\right\|, \quad t \geq 0 .
$$

Moreover, if $A$ is a diagonal stability matrix, then

$$
\left\|E_{\alpha, \beta}\left(A t^{\alpha}\right)\right\| \leq e^{-\omega t}, \quad t \geq 0,
$$

where $-\omega(\omega>0)$ is the largest eigenvalue of the diagonal stability matrix A.
Lemma 6 (see [31]). Let $u(t), a(t)$ be nonnegative and local integrable on $[0, T)(T \leq+\infty)$, and let $g$ be a nonnegative, nondecreasing continuous function defined on $[0, T), g(t) \leq$ $M$, and let $M$ be a real constant, $\alpha>0$, with

$$
u(t) \leq a(t)+g(t) \int_{0}^{t}(t-s)^{\alpha-1} u(s) d s, \quad t \in[0, T) .
$$

Then

$$
\begin{array}{r}
u(t) \leq a(t)+\int_{0}^{t}\left[\sum_{n=1}^{\infty} \frac{(g(t) \Gamma(\alpha))^{n}}{\Gamma(n \alpha)}(t-s)^{n \alpha-1} a(s)\right] d s, \\
t \in[0, T) .
\end{array}
$$

Moreover, if $a(t)$ is a nondecreasing function on $[0, T)$, then

$$
u(t) \leq a(t) E_{\alpha, 1}\left(g(t) \Gamma(\alpha) t^{\alpha}\right), \quad t \in[0, T) .
$$

\section{Main Result}

We first give a key lemma in the proof of our main result as follows.

Lemma 7. Let $u(t), v(t)$ be nonnegative and local integrable on $[0, T)(T \leq+\infty)$, and let $a_{1}(t), a_{2}(t)$ be nonnegative, nondecreasing and local integrable on $[0, T)$, and let $b_{1}, b_{2}$ be two positive constants, $\alpha, \beta>1$, with

$$
\begin{array}{ll}
u(t) \leq a_{1}(t)+b_{1} \int_{0}^{t}(t-s)^{\alpha-1} v(s) d s, & t \in[0, T), \\
v(t) \leq a_{2}(t)+b_{2} \int_{0}^{t}(t-s)^{\beta-1} u(s) d s, & t \in[0, T) .
\end{array}
$$

Then

$$
\begin{aligned}
u(t) \leq & \left(a_{1}(t)+b_{1} \int_{0}^{t}(t-s)^{\alpha-1} a_{2}(s) d s\right) \\
& \times E_{\alpha+\beta}\left(b_{1} b_{2} \Gamma(\alpha) \Gamma(\beta) t^{\alpha+\beta}\right), \quad t \in[0, T), \\
v(t) \leq & \left(a_{2}(t)+b_{2} \int_{0}^{t}(t-s)^{\beta-1} a_{1}(s) d s\right) \\
& \times E_{\alpha+\beta}\left(b_{1} b_{2} \Gamma(\alpha) \Gamma(\beta) t^{\alpha+\beta}\right), \quad t \in[0, T) .
\end{aligned}
$$

Proof. Substituting (32) into (31), we obtain

$$
\begin{aligned}
u(t) \leq & a_{1}(t)+b_{1} \int_{0}^{t}(t-s)^{\alpha-1} v(s) d s \\
\leq & a_{1}(t)+b_{1} \int_{0}^{t}(t-s)^{\alpha-1} a_{2}(s) d s \\
& +b_{1} b_{2} \int_{0}^{t}(t-s)^{\alpha-1} \int_{0}^{s}(s-\xi)^{\beta-1} u(\xi) d \xi .
\end{aligned}
$$


Changing the order of integration in the above double integral, we obtain

$$
\begin{aligned}
u(t) \leq & a_{1}(t)+b_{1} \int_{0}^{t}(t-s)^{\alpha-1} a_{2}(s) d s \\
& +b_{1} b_{2} \int_{0}^{t} u(\xi) d \xi \int_{\xi}^{t}(t-s)^{\alpha-1}(s-\xi)^{\beta-1} d s \\
= & a_{1}(t)+b_{1} \int_{0}^{t}(t-s)^{\alpha-1} a_{2}(s) d s \\
& +b_{1} b_{2} \int_{0}^{t} \frac{\Gamma(\alpha) \Gamma(\beta)}{\Gamma(\alpha+\beta)}(t-\xi)^{\alpha+\beta-1} u(\xi) d \xi
\end{aligned}
$$

Let $a(t)=a_{1}(t)+b_{1} \int_{0}^{t}(t-s)^{\alpha-1} a_{2}(s) d s, g(t)=$ $b_{1} b_{2}((\Gamma(\alpha) \Gamma(\beta)) / \Gamma(\alpha+\beta))$; then $a(t)$ is a nonnegative, nondecreasing, and local integrable function and $g(t)$ is a nonnegative, nondecreasing continuous function. Thus, by Lemma 6 (30), one has

$$
\begin{aligned}
u(t) \leq & \left(a_{1}(t)+b_{1} \int_{0}^{t}(t-s)^{\alpha-1} a_{2}(s) d s\right) \\
& \times E_{\alpha+\beta}\left(b_{1} b_{2} \Gamma(\alpha) \Gamma(\beta) t^{\alpha+\beta}\right) .
\end{aligned}
$$

Similarly, we get

$$
\begin{aligned}
v(t) \leq & \left(a_{2}(t)+b_{2} \int_{0}^{t}(t-s)^{\beta-1} a_{1}(s) d s\right) \\
& \times E_{\alpha+\beta}\left(b_{1} b_{2} \Gamma(\alpha) \Gamma(\beta) t^{\alpha+\beta}\right) .
\end{aligned}
$$

For convenience, let

$$
\begin{array}{r}
R=\sup _{0 \leq s \leq \tau}\{\|R(s)\|\}, \quad P=\sup _{0 \leq s \leq \tau}\{\|P(s)\|\}, \\
h=\max _{1 \leq i \leq n}\left\{h_{i}\right\}, \quad l=\max _{1 \leq j \leq n}\left\{l_{j}\right\}, \\
\Theta(t):=\max \left\{\frac{h}{\alpha} t^{\alpha}\left(1+\frac{t}{\alpha+1}\right)\left(\mu(B)+R \tau e^{\gamma \tau}\right),\right. \\
\left.\frac{l}{\beta} t^{\beta}\left(1+\frac{t}{\beta+1}\right)\left(\mu(D)+P \tau e^{\gamma \tau}\right)\right\},
\end{array}
$$

where $-\gamma$ is the largest eigenvalue of the diagonal stability matrix $-C$ and $\mu(\cdot)$ denotes the largest singular value of matrix $(\cdot)$.
In the following, sufficient conditions for finite-time stability of fractional-order BAM neural networks with distributed delay are derived.

Theorem 8. Let $1<\alpha, \beta<2$. If system (23) satisfies (H1)(H3) with the initial condition (19), and

$$
\begin{aligned}
e^{-\gamma t} & ((1+t)+\Theta(t)) E_{\alpha+\beta} \\
& \times\left[h l\left(\mu(B)+R \tau e^{\gamma \tau}\right)\left(\mu(D)+P \tau e^{\gamma \tau}\right) \Gamma(\alpha) \Gamma(\beta) t^{\alpha+\beta}\right] \\
< & \frac{\varepsilon}{\delta},
\end{aligned}
$$

where $t \in J=[0, T)$, then system (23) is finite-time stable with respect to $\{\delta, \varepsilon, 0, J\}, \delta<\varepsilon$.

Proof. By Laplace transform and inverse Laplace transform, system (23) is equivalent to

$$
\begin{aligned}
u(t)=E_{\alpha}\left(-C t^{\alpha}\right) \bar{\varphi}(0)+t E_{\alpha, 2}\left(-C t^{\alpha}\right) \bar{\varphi}^{\prime}(0) \\
+\int_{0}^{t}(t-s)^{\alpha-1} E_{\alpha, \alpha}\left(-C t^{\alpha}\right) \\
\quad \times[B H(s) v(s) \\
\left.\quad+\int_{0}^{\tau} R(\eta) H(s-\eta) v(s-\eta) d \eta\right] d s,
\end{aligned}
$$

$$
\begin{aligned}
v(t)=E_{\beta}\left(-C t^{\beta}\right) \bar{\psi}(0)+t E_{\beta, 2}\left(-C t^{\beta}\right) \bar{\psi}^{\prime}(0) \\
+\int_{0}^{t}(t-s)^{\beta-1} E_{\beta, \beta}\left(-C t^{\beta}\right) \\
\quad \times[D L(s) u(s) \\
\left.\quad+\int_{0}^{\tau} P(\eta) L(s-\eta) u(s-\eta) d \eta\right] d s .
\end{aligned}
$$

From (40), (41), and Lemma 5, we obtain

$$
\begin{aligned}
\|u(t)\| \leq & \left(\|\bar{\varphi}\|+\left\|\bar{\varphi}^{\prime}\right\| t\right) e^{-\gamma t} \\
& +\int_{0}^{t}(t-s)^{\alpha-1} e^{-\gamma(t-s)} \\
& \times \| B H(s) v(s) \\
& +\int_{0}^{\tau} R(\eta) H(s-\eta) v(s-\eta) d \eta \| d s
\end{aligned}
$$




$$
\begin{aligned}
\|v(t)\| \leq & \left(\|\bar{\psi}\|+\left\|\bar{\psi}^{\prime}\right\| t\right) e^{-\gamma t} \\
& +\int_{0}^{t}(t-s)^{\beta-1} e^{-\gamma(t-s)} \\
& \times \| D L(s) u(s) \\
& \quad+\int_{0}^{\tau} P(\eta) L(s-\eta) u(s-\eta) d \eta \| d s .
\end{aligned}
$$

Let $U(t)=\sup _{\theta \in[t-\tau, t]}\|u(\theta)\| e^{\gamma \theta}$, and $V(t)=$ $\sup _{\theta \in[t-\tau, t]}\|v(\theta)\| e^{\gamma \theta}$; then

$$
\begin{aligned}
\|u(s)\| e^{\gamma s} \leq U(s), & \|u(s-\tau)\| e^{\gamma(s-\tau)} \leq U(s), \\
\|v(s)\| e^{\gamma s} \leq V(s), & \|v(s-\tau)\| e^{\gamma(s-\tau)} \leq V(s) .
\end{aligned}
$$

Thus, we have by (42) and (44) that

$$
\begin{aligned}
& \|u(t)\| e^{\gamma t} \\
& \leq\|\bar{\varphi}\|+\left\|\bar{\varphi}^{\prime}\right\| t \\
& +\int_{0}^{t}(t-s)^{\alpha-1} \\
& \quad \times\left[h \mu(B)\|v(s)\| e^{\gamma s}\right. \\
& \left.\quad+\int_{0}^{\tau} h R\|v(s-\eta)\| e^{\gamma(s-\eta)} e^{\gamma \eta} d \eta\right] d s \\
& \leq\|\bar{\varphi}\|+\left\|\bar{\varphi}^{\prime}\right\| t \\
& +\int_{0}^{t}(t-s)^{\alpha-1} h\left[\mu(B)+R \tau e^{\gamma \tau}\right] V(s) d s,
\end{aligned}
$$

where $\mu(B)$ denotes the largest singular value of matrix $B$. Similarly, by (43) and (45), we get

$$
\begin{aligned}
& \|v(t)\| e^{\gamma t} \\
& \leq\|\bar{\psi}\|+\left\|\bar{\psi}^{\prime}\right\| t \\
& +\int_{0}^{t}(t-s)^{\beta-1} \\
& \quad \times\left[l \mu(D)\|x(s)\| e^{\gamma s}\right. \\
& \left.\quad+\int_{0}^{\tau} l P\|x(s-\eta)\| e^{\gamma(s-\eta)} e^{\gamma \eta} d \eta\right] d s \\
& \leq\|\bar{\psi}\|+\left\|\bar{\psi}^{\prime}\right\| t \\
& +\int_{0}^{t}(t-s)^{\beta-1} l\left[\mu(D)+P \tau e^{\gamma \tau}\right] U(s) d s .
\end{aligned}
$$

Hence, by (46) and (47), we have

$$
\begin{aligned}
U(t) \leq & \|\bar{\varphi}\|_{1}(1+t) \\
& +\int_{0}^{t}(t-s)^{\alpha-1} h\left[\mu(B)+R \tau e^{\gamma \tau}\right] V(s) d s, \\
V(t) \leq & \|\bar{\psi}\|_{1}(1+t) \\
& +\int_{0}^{t}(t-s)^{\beta-1} l\left[\mu(D)+P \tau e^{\gamma \tau}\right] U(s) d s .
\end{aligned}
$$

Set

$$
\begin{array}{cc}
a_{1}(t)=\|\bar{\varphi}\|_{1}(1+t), & a_{2}(t)=\|\bar{\psi}\|_{1}(1+t), \\
b_{1}=h\left(\mu(B)+R \tau e^{\gamma \tau}\right), & b_{2}=l\left(\mu(D)+P \tau e^{\gamma \tau}\right) .
\end{array}
$$

By simple computation, we have

$$
\begin{aligned}
& \int_{0}^{t}(t-s)^{\alpha-1} a_{2}(s) d s \\
& \leq\|\bar{\psi}\|_{1} \int_{0}^{t}(t-s)^{\alpha-1}(1+s) d s \\
&=\frac{\|\bar{\psi}\|_{1}}{\alpha} t^{\alpha}\left(1+\frac{t}{\alpha+1}\right), \\
& \int_{0}^{t}(t-s)^{\beta-1} a_{1}(s) d s \\
& \quad \leq\|\bar{\varphi}\|_{1} \int_{0}^{t}(t-s)^{\beta-1}(1+s) d s \\
& \quad=\frac{\|\bar{\varphi}\|_{1}}{\beta} t^{\beta}\left(1+\frac{t}{\beta+1}\right) .
\end{aligned}
$$

It follows from (48)-(50) and Lemma 7 that

$$
\begin{aligned}
U(t) \leq & {\left[(1+t)\|\bar{\varphi}\|_{1}+\frac{\|\bar{\psi}\|_{1}}{\alpha} t^{\alpha}\right.} \\
& \left.\times\left(1+\frac{t}{\alpha+1}\right) h\left(\mu(B)+R \tau e^{\gamma \tau}\right)\right] \\
& \cdot E_{\alpha+\beta}\left[h l\left(\mu(B)+R \tau e^{\gamma \tau}\right)\right. \\
V(t) \leq & \left.\quad \times\left(\mu(D)+P \tau e^{\gamma \tau}\right) \Gamma(\alpha) \Gamma(\beta) t^{\alpha+\beta}\right], \\
& \left.\times\left(1+\frac{t}{\beta+1}\right) l\left(\mu(D)+P \tau e^{\gamma \tau}\right)\right] \\
& \cdot E_{\alpha+\beta}\left[h l\left(\mu(B)+R \tau \|^{\gamma \tau}\right)\right. \\
& \left.\times\left(\mu(D)+P \tau e^{\gamma \tau}\right) \Gamma(\alpha) \Gamma(\beta) t^{\alpha+\beta}\right] .
\end{aligned}
$$


By (51), we obtain

$$
\begin{aligned}
& \|(u(t), v(t))\| \\
& =\|u(t)\|+\|v(t)\| \\
& \leq e^{-\gamma t}\|(\bar{\varphi}, \bar{\psi})\|_{1}((1+t)+\Theta(t)) \\
& \quad \times E_{\alpha+\beta}\left[h l\left(\mu(B)+R \tau e^{\gamma \tau}\right)\right. \\
& \left.\quad \times\left(\mu(D)+P \tau e^{\gamma \tau}\right) \Gamma(\alpha) \Gamma(\beta) t^{\alpha+\beta}\right] .
\end{aligned}
$$

Thus, if condition (39) is satisfied and $\|(\bar{\varphi}, \bar{\psi})\|_{1}<\delta$, then $\|(u(t), v(t))\|<\varepsilon, t \in J$; that is, system (23) is finite-time stable. This completes the proof.

\section{An Illustrative Example}

In this section, we give an example to illustrate the effectiveness of our main result.

Consider the following two-state Caputo fractional BAM type neural networks model with distributed delay

$$
\begin{aligned}
& { }_{0}^{C} D_{t}^{\alpha} x_{1}(t)=-0.7 x_{1}(t)-0.2 f_{1}\left(y_{1}(t)\right)+0.1 f_{2}\left(y_{2}(t)\right) \\
& +\int_{0}^{\tau} s^{3 / 2} f_{1}\left(y_{1}(t-s)\right) d s \\
& +\int_{0}^{\tau} s f_{2}\left(y_{2}(t-s)\right) d s \\
& { }_{0}^{C} D_{t}^{\alpha} x_{2}(t)=-0.6 x_{2}(t)+0.3 f_{1}\left(y_{1}(t)\right)+0.2 f_{2}\left(y_{2}(t)\right) \\
& +\int_{0}^{\tau} s f_{1}\left(y_{1}(t-s)\right) d s \\
& -\int_{0}^{\tau} s^{3 / 2} f_{2}\left(y_{2}(t-s)\right) d s \\
& { }_{0}^{C} D_{t}^{\beta} y_{1}(t)=-0.7 y_{1}(t)+0.4 g_{1}\left(x_{1}(t)\right)+0.2 g_{2}\left(x_{2}(t)\right) \\
& -\int_{0}^{\tau} s g_{1}\left(x_{1}(t-s)\right) d s \\
& +\int_{0}^{\tau} s^{2} g_{2}\left(x_{2}(t-s)\right) d s \\
& { }_{0}^{C} D_{t}^{\beta} y_{2}(t)=-0.6 y_{2}(t)+0.1 g_{1}\left(x_{1}(t)\right)-0.3 g_{2}\left(x_{2}(t)\right) \\
& +\int_{0}^{\tau} s^{2} g_{1}\left(x_{1}(t-s)\right) d s \\
& +\int_{0}^{\tau} s g_{2}\left(x_{2}(t-s)\right) d s
\end{aligned}
$$

with the initial condition

$$
\begin{aligned}
x(t)=\varphi(t)=\frac{1}{15} \sin t, \quad x^{\prime}(t)=\varphi^{\prime}(t) & =\frac{1}{15} \cos t, \\
t & \in[-\tau, 0], \\
y(t)=\psi(t)=\frac{1}{15} \cos t, \quad y^{\prime}(t)=\psi^{\prime}(t) & =-\frac{1}{15} \sin t, \\
t & \in[-\tau, 0],
\end{aligned}
$$

where $\alpha=1.2, \beta=1.3, \tau=0.2$, and $f_{j}\left(x_{j}\right)=g_{j}\left(x_{j}\right)=$ $(1 / 2)\left(\left|x_{j}+1\right|-\left|x_{j}-1\right|\right), j=1,2$. It is easy to know that $\left(x_{1}^{*}, x_{2}^{*}, y_{1}^{*}, y_{2}^{*}\right)^{T}=(0,0,0,0)^{T}$ is an equilibrium point of system (53). Since $\|(\varphi, \psi)\|_{1}=1 / 15<0.07$, we may let $\delta=0.07$. Take

$$
\begin{gathered}
t_{0}=0, \quad J=[0,30), \quad \varepsilon=1, \\
C=\left[\begin{array}{cc}
-0.7 & 0 \\
0 & -0.6
\end{array}\right], \quad B=\left[\begin{array}{cc}
-0.2 & 0.1 \\
0.3 & 0.2
\end{array}\right], \\
D=\left[\begin{array}{cc}
0.4 & 0.2 \\
0.1 & -0.3
\end{array}\right], \quad R(s)=\left[\begin{array}{cc}
s^{3 / 2} & s \\
s & -s^{3 / 2}
\end{array}\right], \\
P(s)=\left[\begin{array}{cc}
-s & s^{2} \\
s^{2} & s
\end{array}\right] .
\end{gathered}
$$

It is easy to check that

$$
\begin{gathered}
h=l=1, \quad \gamma=0.6, \quad \mu(B)=0.3828, \\
\mu(D)=0.4515, \quad R=0.2894, \quad P=0.24, \\
\Theta(t)=\max \left\{0.1697 t^{1.2}(t+2.2), 0.1691 t^{1.3}(t+2.3)\right\}, \\
E_{\alpha+\beta}\left[h l\left(\mu(B)+R \tau e^{\gamma \tau}\right)\left(\mu(D)+P \tau e^{\gamma \tau}\right) \Gamma(\alpha) \Gamma(\beta) t^{\alpha+\beta}\right] \\
=E_{2.5}\left(0.1867 t^{2.5}\right) .
\end{gathered}
$$

From condition (41) of Theorem 8, we can get

$$
\begin{gathered}
e^{-0.6 t}[(1+t) \\
+\max \left\{0.1697 t^{1.2}(t+2.2),\right. \\
\left.\left.0.1691 t^{1.3}(t+2.3)\right\}\right] \\
\times E_{2.5}\left(0.1867 t^{2.5}\right)<\frac{1}{0.07} .
\end{gathered}
$$

We can obtain that the estimated time of finite-time stability is $T \approx 23.78$. Hence, system (53) is finite-time stable with respect to $\{0.07,1,0,[0,30)\}$.

\section{Conflict of Interests}

The authors declare that there is no conflict of interests regarding the publication of this paper. 


\section{Acknowledgments}

This work is supported by the Natural Science Foundation of Jiangsu Province (BK2011407) and the Natural Science Foundation of China (11271364 and 10771212).

\section{References}

[1] K. S. Miller and B. Ross, An Introduction to the Fractional Calculus and Fractional Differential Equations, John Wiley \& Sons, New York, NY, USA, 1993.

[2] I. Podlubny, Fractional Differential Equations, Academic Press, San Diego, Calif, USA, 1999.

[3] E. Soczkiewicz, "Application of fractional calculus in the theory of viscoelasticity," Molecular and Quantum Acoustics, vol. 23, pp. 397-404, 2002.

[4] V. V. Kulish and J. L. Lage, "Application of fractional calculus to fluid mechanics," Journal of Fluids Engineering, vol. 124, no. 3, pp. 803-806, 2002.

[5] A. A. Kilbas, H. M. Srivastava, and J. J. Trujillo, Theory and Applications of Fractional Differential Equations, vol. 204 of North-Holland Mathematics Studies, Elsevier Science, Amsterdam, The Netherlands, 2006.

[6] J. Sabatier, O. P. Agrawal, and J. Machado, Theoretical Developments and Applications, Advance in Fractional Calculus, Springer, Berlin, Germany, 2007.

[7] P. Arena, R. Caponetto, L. Fortuna, and D. Porto, "Bifurcation and chaos in noninteger order cellular neural networks," International Journal of Bifurcation and Chaos in Applied Sciences and Engineering, vol. 8, no. 7, pp. 1527-1539, 1998.

[8] P. Arena, L. Fortuna, and D. Porto, "Chaotic behavior in noninteger-order cellular neural networks," Physical Review E: Statistical Physics, Plasmas, Fluids, and Related Interdisciplinary Topics, vol. 61, no. 1, pp. 776-781, 2000.

[9] M. P. Lazarević, "Finite time stability analysis of $P D^{\alpha}$ fractional control of robotic time-delay systems," Mechanics Research Communications, vol. 33, no. 2, pp. 269-279, 2006.

[10] A. Boroomand and M. Menhaj, "Fractional-order Hopfield neural networks," in Proceedings of the Natural Computation International Conference, pp. 883-890, 2010.

[11] E. Kaslik and S. Sivasundaram, "Dynamics of fractional-order neural networks," in Proceedings of the International Joint Conference on Neural Network (IJCNN '11), pp. 611-618, August 2011.

[12] E. Kaslik and S. Sivasundaram, "Nonlinear dynamics and chaos in fractional-order neural networks," Neural Networks, vol. 32, pp. 245-256, 2012.

[13] R. Zhang, D. Qi, and Y. Wang, "Dynamics analysis of fractional order three-dimensional Hopfield neural network," in Proceedings of the 6th International Conference on Natural Computation (ICNC '10), pp. 3037-3039, August 2010.

[14] H. Delavari, D. Baleanu, and J. Sadati, "Stability analysis of Caputo fractional-order nonlinear systems revisited," Nonlinear Dynamics, vol. 67, no. 4, pp. 2433-2439, 2012.

[15] Y. Li, Y. Chen, and I. Podlubny, "Mittag-Leffler stability of fractional order nonlinear dynamic systems," Automatica, vol. 45, no. 8, pp. 1965-1969, 2009.

[16] Y. Li, Y. Chen, and I. Podlubny, "Stability of fractional-order nonlinear dynamic systems: lyapunov direct method and generalized Mittag-Leffler stability," Computers \& Mathematics with Applications, vol. 59, no. 5, pp. 1810-1821, 2010.
[17] K. Gu, V. L. Kharitonov, and J. Chen, Stability of Time-delay Systems, Birkhäuser, Boston, Mass, USA, 2003.

[18] S. Arik, "An analysis of global asymptotic stability of delayed cellular neural networks," IEEE Transactions on Neural Networks, vol. 13, no. 5, pp. 1239-1242, 2002.

[19] J. Tian, W. Xiong, and F. Xu, "Improved delay-partitioning method to stability analysis for neural networks with discrete and distributed time-varying delays," Applied Mathematics and Computation, vol. 233, pp. 152-164, 2014.

[20] J. Tian, Y. Li, J. Zhao, and S. Zhong, "Delay-dependent stochastic stability criteria for Markovian jumping neural networks with mode-dependent time-varying delays and partially known transition rates," Applied Mathematics and Computation, vol. 218, no. 9, pp. 5769-5781, 2012.

[21] L. Chen, Y. Chai, R. Wu, and T. Zhai, "Dynamic analysis of a class of fractional-order neural networks with delay," Neurocomputing, vol. 111, pp. 190-194, 2013.

[22] R.-C. Wu, X.-D. Hei, and L.-P. Chen, "Finite-time stability of fractional-order neural networks with delay," Communications in Theoretical Physics, vol. 60, no. 2, pp. 189-193, 2013.

[23] A. Alofi, J. Cao, A. Elaiw, and A. Al-Mazrooei, "Delaydependent stability criterion of caputo fractional neural networks with distributed delay," Discrete Dynamics in Nature and Society, vol. 2014, Article ID 529358, 6 pages, 2014.

[24] J. J. Hopfield, "Neurons with graded response have collective computational properties like those of two-state neurons," Proceedings of the National Academy of Sciences of the United States of America, vol. 81, no. 10, pp. 3088-3092, 1984.

[25] B. Kosko, "Bidirectional associative memories," IEEE Transactions on Systems, Man, and Cybernetics, vol. 18, no. 1, pp. 49-60, 1988.

[26] S. Arik and V. Tavsanoglu, "Global asymptotic stability analysis of bidirectional associative memory neural networks with constant time delays," Neurocomputing, vol. 68, no. 1-4, pp. 161176, 2005.

[27] S. Senan, S. Arik, and D. Liu, "New robust stability results for bidirectional associative memory neural networks with multiple time delays," Applied Mathematics and Computation, vol. 218, no. 23, pp. 11472-11482, 2012.

[28] B. Liu, "Global exponential stability for BAM neural networks with time-varying delays in the leakage terms," Nonlinear Analysis: Real World Applications, vol. 14, no. 1, pp. 559-566, 2013.

[29] R. Raja and S. M. Anthoni, "Global exponential stability of BAM neural networks with time-varying delays: the discretetime case," Communications in Nonlinear Science and Numerical Simulation, vol. 16, no. 2, pp. 613-622, 2011.

[30] M. de la Sen, "About robust stability of Caputo linear fractional dynamic systems with time delays through fixed point theory," Fixed Point Theory and Applications, vol. 2011, Article ID 867932, 19 pages, 2011.

[31] H. Ye, J. Gao, and Y. Ding, "A generalized Gronwall inequality and its application to a fractional differential equation," Journal of Mathematical Analysis and Applications, vol. 328, no. 2, pp. 1075-1081, 2007. 


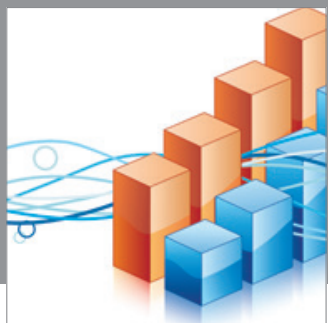

Advances in

Operations Research

mansans

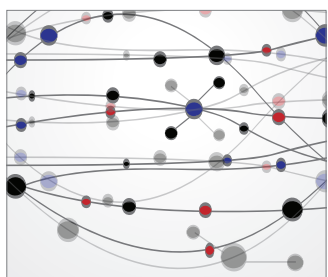

The Scientific World Journal
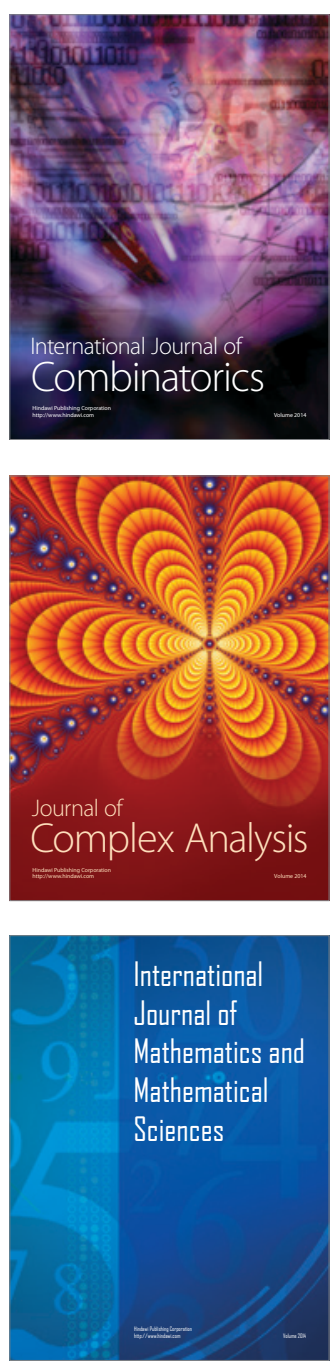
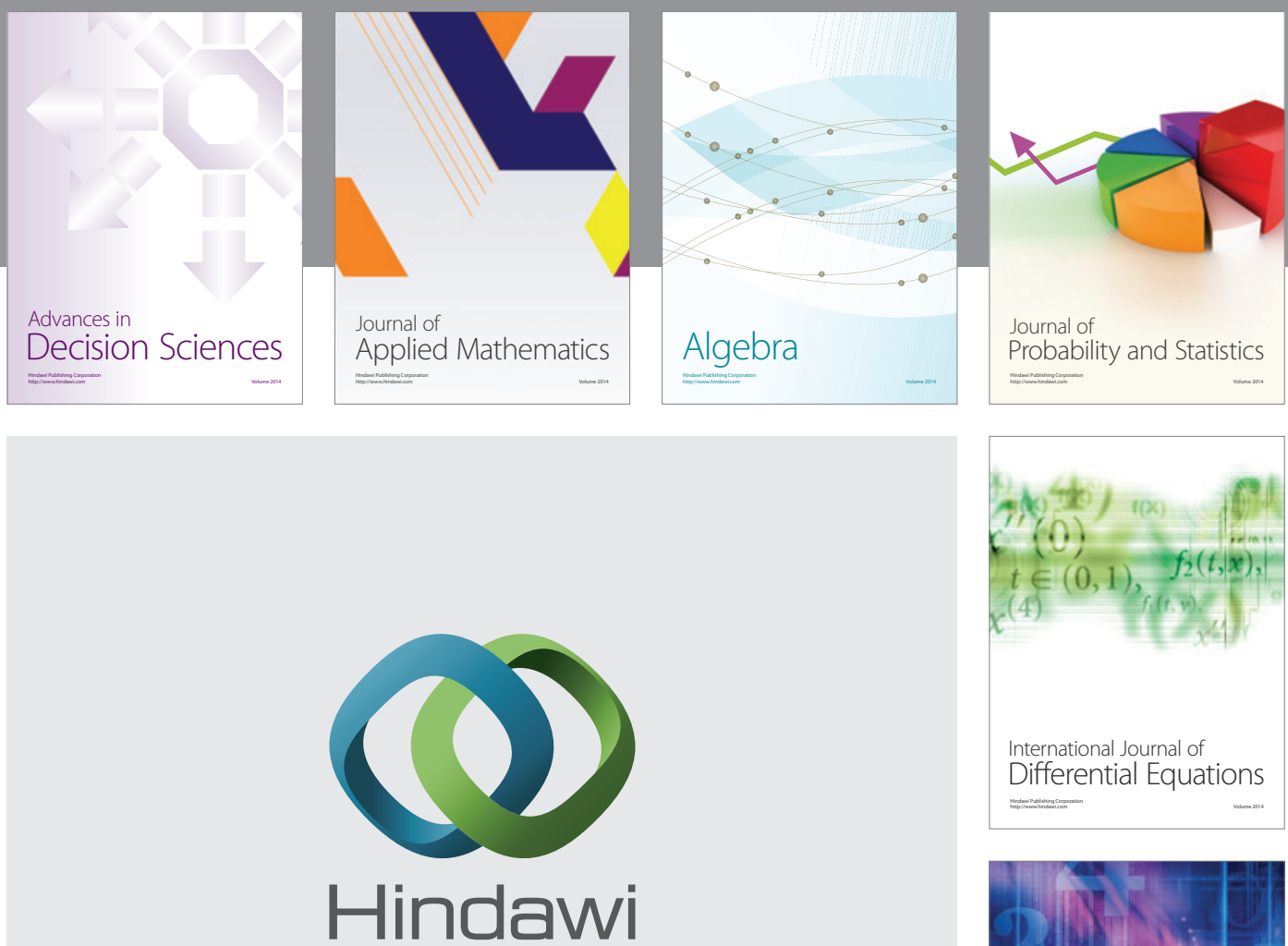

Submit your manuscripts at http://www.hindawi.com
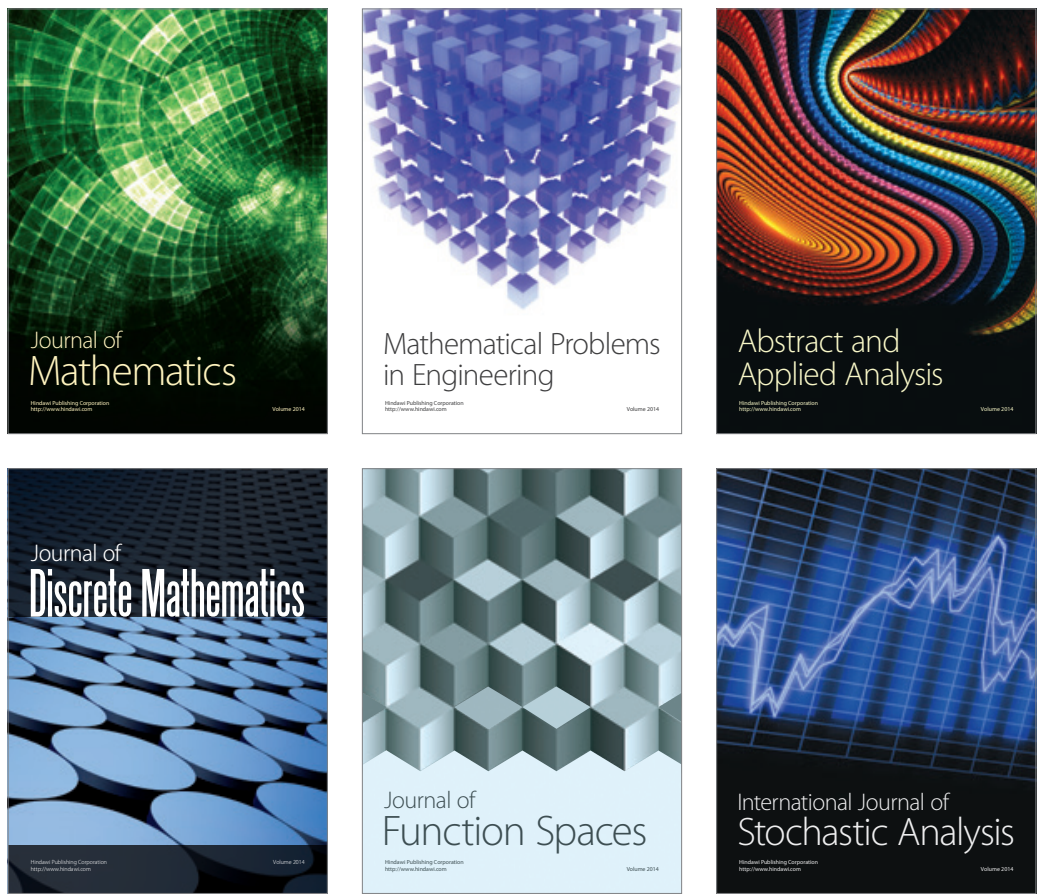

Journal of

Function Spaces

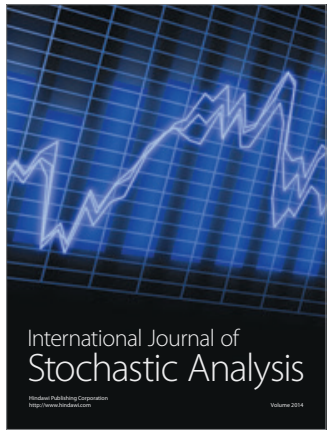

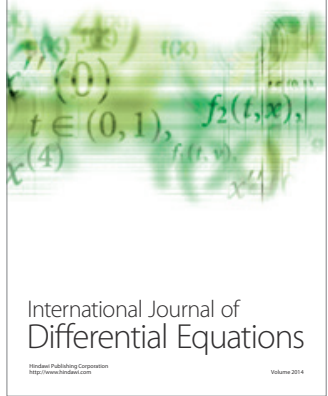
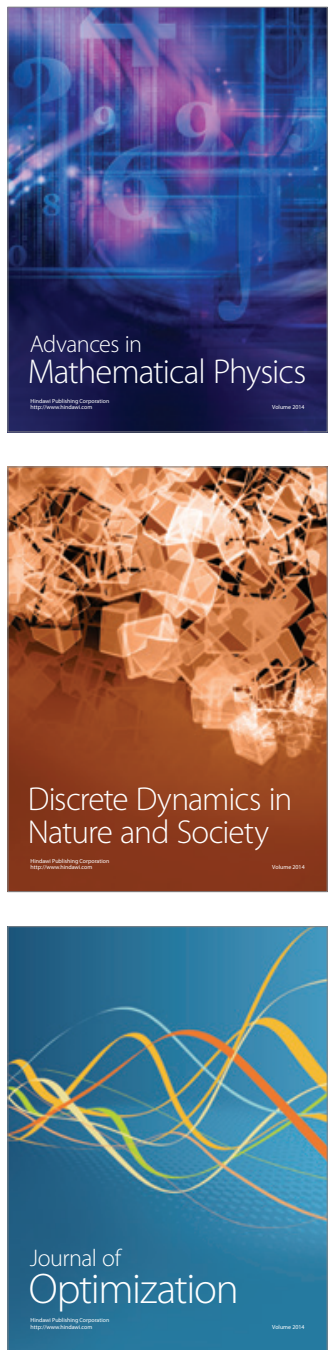